\title{
Análisis sobre las competencias mediacionales que posee el tutor virtual de la UNED de Costa Rica
}

Yahaira Gamboa Villalobos ${ }^{1} \&$ Francisco Mora Vicarioli²

1. Universidad Estatal a Distancia, (UNED). Costa Rica; ygamboa@uned.ac.cr 2. Universidad Estatal a Distancia, (UNED). Costa Rica; fmora@uned.ac.cr

Recibido: 30 de setiembre del 2014

Corregido: 25 de julio del 2015

Aceptado: 03 de agosto del 2015

\begin{abstract}
Resumen
El tutor a distancia, en particular el que se desempeña con apoyo de los entornos virtuales de aprendizaje, debe asumir un rol de asesor pedagógico que oriente y guíe al estudiantado hacia el éxito en el modelo a distancia, esto por medio de diversas funciones, muchas relativas a la comunicación que se ejerce con apoyo de estos espacios virtuales y que permiten incentivar un papel más activo de los estudiantes. En este artículo se expone los resultados de un estudio cuantitativo que perseguían conocer el nivel de capacidades tecno-pedagógicas con los que cuentan los tutores de la Universidad Estatal a Distancia (UNED) de Costa Rica. De este estudio se desprende como se realiza la mediación de algunos cursos en línea por parte del profesorado, desde el inicio del mismo, uso de herramientas de comunicación, evaluación de las actividades y realimentación, de lo anterior se realizan importantes hallazgos que permiten caracterizar las prácticas utilizadas por parte la población estudiada.
\end{abstract}

Palabras claves: educación a distancia, aprendizaje a distancia, aprendizaje virtual, estudiante universitario, universidad a distancia.

\section{Abstract \\ Analysis of the mediational competencies held by the virtual tutor at UNED in Costa Rica}

The distance tutor, particularly that serves to support virtual learning environments, should assume a role of pedagogical advisor to guide and lead the students to success in the remote model, this through a variety of functions, many relating to communication exercised with the support of these virtual spaces and allow encourage a more active role of students. In this article the results of a quantitative study chasing know the level of techno-pedagogical skills at their disposal tutors State University (UNED) in Costa Rica is exposed. This study shows how the mediation of some online courses by teachers is done from the beginning thereof, use of communications tools, assessment and feedback activities of the above major findings are made that allow characterize the practices used by the study population.

Key words: distance education, distance learning, virtual learning, college student, college distance.

\section{INTRODUCCIÓN}

El tutor y las funciones que este ejerce en los cursos a distancia con algún componente virtual son de vital importancia, esto porque es necesario cumplir con aspectos como: comunicación, evaluación, seguimiento, orientación, entre otros que son claves para el correcto aprendizaje del estudiante. Este tipo de modalidad se va expandiendo dado el uso de las tecnologías, sobre esto Ramírez y Rama (2014), señalan que

En la última década, la educación a distancia en América Latina se ha ampliado debido al desarrollo de los programas informáticos y otros proyectos de software propietario para el aprendizaje en línea. Este modelo de enseñanza se ha convertido en un mecanismo de integración y de democratización. Promueve el derecho a la educación permanente 
con igualdad de oportunidades, sin límites de espacio, tiempo, edad, sexo o realidad laboral (Ramírez y Rama, 2014, p.104).

Aunado a lo anterior, la educación a distancia promueve un mejor acceso, dado que:

Elimina las barreras geográficas y facilita el acceso a la educación, independientemente de donde residan incluso personas adultas con estudios postergados. También proporciona flexibilidad en el horario, ya que no hay hora exacta para acceder a la información, lo cual facilita la organización del tiempo personal del estudiante, respetando la vida familiar, social y laboral (Domínguez y Rodríguez, 2013, p. 49).

Precisamente el hecho de que exista un crecimiento de la oferta a distancia a la región responsabiliza por la supervisión de la calidad de los cursos y el rol que ejerce el tutor virtual en ellos. El hecho de detectar posibles carencias permite brindar recomendaciones en cuanto a la tutoría a distancia que se realiza en los medios electrónicos. El objetivo de esta investigación fue identificar el nivel de capacitación y funciones que ejercen los tutores de la UNED de Costa Rica en el área tecno-pedagógica, en especial en el uso de las plataformas virtuales educativas.

El Programa de Aprendizaje en Línea (PAL) de la UNED, se encarga de asesorar a los tutores en el montaje y el desarrollo de cursos con algún componente virtual o bien completamente virtuales, en este quehacer diario los asesores atienden diferentes medios de comunicación donde se plantean las consultas de los estudiantes: correo electrónico, teléfono y página en la red social Facebook, con los cuales, se ha detectado una deficiencia en las competencias de muchos de estos tutores, situación que preocupa, porque la UNED es una universidad a distancia y gran parte de sus cursos se desarrollan con ayuda de las plataformas virtuales educativas. De acuerdo a lo anterior, es que se visualiza la importancia de conocer con detalle cuál es el nivel de competencias en cuanto a la mediación que manejan los tutores de la UNED de Costa Rica para buscar las soluciones necesarias con el fin de fortalecer a quienes son la cara de la universidad, a la vez que se brindan algunas recomendaciones para la mejora de estos procesos académicos.

\section{— Virtualización en la UNED}

En la UNED existen diferentes grados de virtualidad o niveles de uso de las plataformas de aprendizaje en línea, estos se encuentran definidos en el documento denominado "Cómo diseñar y ofertar cursos en línea en tres niveles a saber: nivel básico, intermedio y avanzado" (UNED, 2010). Los distintos grados de virtualidad implican una función distinta para el profesorado y, en consecuencia, una diferencia en el tiempo que se debe invertir en la atención del curso. A continuación se explican estos tres niveles:

- El nivel básico de virtualidad se refiere a cursos en que no existen actividades evaluadas, sino que el entorno se utiliza como repositorio de recursos y prácticas; además, se le confiere la función de un foro para enviar consultas acerca de los contenidos del curso.

- El nivel intermedio incluye evaluación sumativa, así como contenidos y canales de comunicación; pero no se completa la totalidad de la evaluación del curso: más bien es un recurso de apoyo o complementario.

- El nivel avanzado engloba los cursos que son totalmente, en los que, de hecho, existe un alto grado de interacción y actividades. Igualmente, se encuentran recursos y canales de comunicación y, por tanto, el curso se desarrolla por completo en la plataforma virtual.

El tipo de oferta de curso en la UNED conlleva el uso de los términos curso bimodal o curso híbrido. El primero se refiere a un curso en el cual el estudiante selecciona si realiza actividades en la plataforma virtual o no. El curso híbrido es aquel que ofrece actividades en línea y otras evaluadas en forma presencial como exámenes y entrega de tareas en los diversos centros universitarios a lo largo y ancho del país.

Los grados de virtualidad y el tipo de oferta de curso permiten contar con una visión más clara de la variedad de asignaturas de la UNED; las plataformas 
virtuales se aprovechan de distintas formas y existe incluso la opción de que el estudiante las utilice o no.

\section{El tutor virtual, su rol y funciones}

Las funciones del tutor virtual son muchas, dadas las posibilidades de comunicación y las actividades que se pueden desarrollar en un curso en línea, el hecho de conocer tales funciones permite que el docente las ejecute correctamente en el curso y las vaya incorporando de manera paulatina.

Sobre el papel del tutor virtual, se destaca lo señalado por Arboleda y Rama (2013):

En términos esenciales y estructurales, el rol del docente en la modalidad de educación a distancia, cualquiera sea su metodología y mediaciones, no se centra en la transmisión directa de información al estudiante en determinados espacios y tiempos coincidentes, sino en la planificación pedagógica de contenidos orientados a la gestión de conocimientos por parte del estudiante, su acompañamiento, seguimiento y realimentación de sus resultados (p. 107).

Por su parte Delauro (2011), aporta sobre este profesional que:

En líneas generales, podemos sintetizar que las tareas típicas de todo tutor que se desempeña en un entorno virtual son las siguientes: manejo de las herramientas del entorno donde desenvuelve su labor; seguimiento de los procesos de enseñanza y aprendizaje; comunicación sobre los problemas o dificultades; mediación entre las discusiones de los cursantes y entre estos y los contenidos (Delauro 2011, p.12).

Analizando los aportes de los autores anteriores, se hace notable que el rol de un tutor virtual se centra en mediar, acompañar y orientar a sus estudiantes dentro de su proceso de enseñanza aprendizaje.

A continuación se destacan aquellas funciones del tutor virtual que, por su aplicación práctica y por su relación con la comunicación y mediación, deben tenerse en cuenta a la hora de trabajar en un curso en línea. Así mismo, estas pueden influir en la dinámica y la participación en las distintas actividades y evitar posibles conflictos.

\section{- Canales de comunicación}

Mantener los canales de comunicación abiertos significa poner a disposición de todos, una variedad de herramientas de comunicación y usarlas de manera adecuada así como constante.

En los cursos en línea, se sugiere el uso del correo interno y mantener durante todo el curso un foro de consultas. Estos son espacios que permiten que las y los estudiantes envíen mensajes personalizados al tutor o publicar consultas visibles para todo el grupo.

\section{Atención de consultas}

Es necesario brindar respuestas oportunas y precisas a consultas planteadas en foros, correo interno o cualquier otro espacio de comunicación donde las y los estudiantes realicen sus consultas.

Para el caso de las repuestas en foros de consultas o en correo interno, se ha determinado en la UNED que el plazo máximo de 24 horas es el idóneo, esto basado en la experiencia de los Productores Académicos del Programa de Aprendizaje en Línea, aunque a nivel del Reglamento General Estudiantil de la UNED establece en 48 horas, dicho plazo. Así, el estudiante puede resolver su inquietud y proseguir con sus actividades sin que exista atraso por falta de respuesta del facilitador.

Es muy importante también aclarar desde el inicio del curso el plazo en que se resolverán las consultas para que no necesariamente se esperen respuestas en un plazo inferior a 24 horas aunque sí sea factible. Sin embargo, no debe implicar que el profesor deba revisar varias veces por día los espacios de comunicación del curso, lo cual a veces resulta complejo.

\section{- Comunicación para cursos en línea}

Los cursos en línea requieren consideraciones particulares: el hecho de contar con un curso implementado 
en plataforma virtual, una correcta selección de actividades, evaluación, contenidos o recursos, distribución visual y diseño gráfico, entre otros elementos, no garantiza para nada el éxito del curso ni que vaya a resultar una experiencia de aprendizaje idónea para el estudiantado, sino se cuidan también otros detalles durante su ejecución.

La correcta comunicación y mediación en la tutoría virtual permiten brindar un mejor acompañamiento durante el proceso, pues se relacionan con la forma en que se atiende al estudiantado en los diferentes espacios de comunicación e interacción y se brinda realimentación en las distintas actividades que puede haber en un curso.

\section{- Evaluación y realimentación}

Ofrecer una realimentación oportuna a las y los estudiantes sobre la evaluación de los aprendizajes permite orientarle sobre los puntos que son necesarios mejorar para su correcto aprendizaje.

Tal acción se lleva a cabo preferiblemente por medio de instrumentos de evaluación diseñados para tal fin. Este debe relacionarse con lo que se solicita en las consignas de la actividad; también son útiles los comentarios generales que el profesorado le brinda al estudiante acerca de los elementos de forma y contenido que deben ser mejorados en las actividades evaluadas.

El hecho de presentar una realimentación oportuna beneficia a las y los estudiantes pues les permite conocer su avance con respecto a la evaluación, los objetivos y los contenidos del curso. Es beneficio que la evaluación en la modalidad virtual se entregue siete días naturales luego de la fecha límite de entrega o finalización de la actividad, aunque en el Reglamento General Estudiantil de la UNED se consigna en 15 días naturales, pues se basa en el periodo normal de los instrumentos de evaluación físicos.

Como se indica en el Reglamento General Estudiantil de la UNED (2012), los procesos evaluativos cumplen dos funciones primordiales:

a. Informar a cada estudiante del progreso en su aprendizaje, determinar cuáles de ellos ha interiorizado los objetivos y las competencias establecidas para otorgar la certificación que ofrece el plan de estudios.

b. Conocer y entender los logros y dificultades que experimenta cada estudiante, con el fin de introducir los cambios necesarios para que aprenda de forma significativa y sea capaz de autorregular su propio aprendizaje (p.27).

Estas funciones de los procesos evaluativos destacan la importancia de la realimentación pronta al estudiante, pues permite que este conozca su avance y el nivel de logro con los objetivos que se deben alcanzar.

\section{- Armonía}

Mantener la armonía y la sana interacción entre los participantes del curso se puede lograr por medio de mensajes oportunos y una comunicación adecuada en los diferentes espacios.

La armonía entre los participantes implica una revisión constante de los espacios de comunicación para detectar cualquier anomalía o situación irregular. Desde el inicio, se debe normar este tema en el curso. Para ello, se recomienda aplicar las normas de la netiquetas (serie de reglas orientadas hacia el bueno uso de los medios digitales en cuanto a la comunicación), que promueven una relación respetuosa y ordenada entre los participantes.

El PAL cuenta con un documento referido a las reglas de la netiqueta, denominado Directrices para el trabajo en la plataforma, el cual se recomienda dirigir a las y los estudiantes con el fin de que conozcan la correcta comunicación en el curso.

Por otro lado, los trabajos grupales o colaborativos que se desarrollan en el curso deben ser monitoreados; de esta forma, se pueden percibir conflictos o malos entendidos y realizar intervenciones a oportuna. Además, el profesor debe tener en cuenta que el Reglamento General Estudiantil de la UNED, en su artículo 27, penaliza el uso impropio del vocabulario.

\section{- Información}

Es necesario mantener informados a las y los estudiantes en todo momento: hay que avisarles las 
novedades del curso, algún cambio o una aclaración que se realice en alguna actividad. Desde luego, se pueden utilizar las distintas herramientas que proveen las plataformas de aprendizaje en línea:

a. Herramienta de anuncios: en el caso de la plataforma Blackboard, consiste en un recurso informativo que se coloca en el menú izquierdo del curso y se reflejan las publicaciones en el bloque de Novedades (página de inicio); así mismo, se cuenta con la posibilidad de enviar una copia del anuncio al correo personal de los participantes. En la plataforma de aprendizaje en línea Moodle se puede utilizar el bloque denominado HTML para tal fin.

b. Calendario: ese trata de una herramienta que posee tanto la plataforma Moodle como Blackboard y tiene la posibilidad de colocar los eventos que sean necesarios para propiciar una mayor organización por parte de las y los estudiantes con respecto de las actividades que se concretan en el curso.

c. Correo interno: la herramienta sirve para hacer indicaciones de manera mucho más personalizada. Tanto Blackboard como Moodle habilitan el correo interno y permite el control de situaciones particulares con los estudiantes. El medio permite también el envío de archivos adjuntos.

\section{- Presencia}

Mantener y reflejar la permanencia en el entorno virtual brinda la sensación de mayor cercanía con las y los estudiantes quienes se sentirán más acompañados durante el proceso a distancia. Cumplir con las recomendaciones mencionadas como la actualización de los recordatorios, los diversos avisos y la mediación constante en los espacios de interacción, son aspectos que orientan a una buena presencia dentro del curso.

Se recomienda revisar todos los días el curso virtual, en aras de cumplir con plazos de respuesta a inquietudes planteadas por el estudiantado.

\section{- Revisión del ingreso de los estudiantes}

Al inicio del curso el profesorado debe estar pendiente del acceso oportuno de las y los estudiantes al entorno. Luego de que transcurre la primera semana se debe revisar el registro de la plataforma para detectar cuáles estudiantes no han realizado el ingreso al curso. Entonces se puede tratar de contactar a las y los estudiantes vía telefónica para recordarles del inicio del curso y para verificar si existieron problemas con los datos de acceso o de otra índole.

Cuando lo anterior no se hace, es probable que el estudiante nunca logre ingresar o que se integre ya tarde al curso y pierda actividades evaluadas. Igualmente, cuando las y los estudiantes no están presentes desde el inicio del proceso del curso se les puede dificultar.

\section{Cierre de actividades y solución de tareas}

Cuando finalizan los foros de discusión, las wikis, los blogs o las tareas, es adecuado brindar un cierre o solución porque permite llegar a puntos en común y rectificar posibles errores cometidos. Tal actividad responde a la dimensión formativa de la evaluación de los aprendizajes. También esta se puede designar a las y los estudiantes que no hayan tenido buena participación en las actividades, de tal forma que puedan cumplir adecuadamente con los objetivos de aprendizaje.

\section{- Organización}

Uno de los roles de gran peso de un tutor virtual es la organización de su curso y entre algunas de las funciones que debe cumplir se destacan: definir cronogramas de trabajo, fechas y horas de entrega de las diferentes actividades desde el inicio. Puntos fundamentales dentro de la educación a distancia que incentivan en las y los estudiantes una mejor organización de su tiempo y por ende a ser autodidactas.

\section{- Dinamizador}

La función dinamizadora que debe cumplir el tutor virtual se relaciona con las intervenciones en espacios de interacción que motivan las participaciones de los estudiantes, el planteamiento de preguntas de análisis en los foros, los recordatorios cuando se percibe 
falta de participación y los recordatorios de entregas en caso de que existan estudiantes que no las hayan llevado a cabo.

En los trabajos colaborativos como los que se desarrollan en las wikis, el profesorado debe revisar constantemente el avance grupal y realizar recordatorios por medio del correo interno o anuncios, esto con el fin de incentivar la participación grupal.

\section{METODOLOGÍA}

La metodología empleada consistió en la aplicación de un instrumento distribuido de forma electrónica (por medio de correo electrónico) al personal que se ha desempeñado como tutores virtuales. No se utilizó ningún tipo de muestra específica, sino que la misma fue definida a conveniencia, en total se distribuyó la encuesta a 71 tutores y fue respondida por 45, esto para una colaboración del $63 \%$. Cabe señalar que el instrumento estaba constituido en los siguientes apartados:

1. Preguntas administrativas (conformado por cuatro preguntas),

2. Quehaceres de un tutor virtual (conformado por 24 ítems), subdivido en:

2.1 Proceso inicial del curso (conformado por diez ítems),

2.2 Desarrollo del curso (conformado por 12 ítems) y

2.3 Evaluación del curso (conformado por tres ítems).

3. Apoyo de la institución para su labor como tutor (conformado por cinco ítems).

El instrumento tenía un total de 54 preguntas relacionadas con el quehacer del tutor virtual, sobre el tipo de capacitación y apoyo que ha brindado la institución para tal fin.

El estudio fue de corte cuantitativo por el tipo de ítems que se utilizaron, lo que permitió un adecuado análisis de las categorías propuestas, las cuales se analizan a continuación.

\section{RESULTADOS}

A continuación los aspectos más relevantes obtenidos por medio de la encuesta que se aplicó a tutores de la UNED que han impartido cursos en línea.

Iniciando con los datos generales se obtuvo que la mayoría de los tutores encuestados pertenece a la Escuela de Ciencias de la Educación (ECE) y a la Escuela de Ciencias Sociales y Humanidades $(E S C H)$ y en tercer orden de cantidad se encuentran los de la Escuela de Ciencias Exactas y Naturales (ECEN), los resultados se presentan en el Figura 1.

En cuanto al tiempo de laborar en la UNED el $45 \%$ de los tutores tienen más de seis años y trabajan tiempo completo (39\%), esto se puede apreciar en el Figura 2, en relación a la jornada laboral, la mayoría de tutores son de cuarto de tiempo y de un cuarto de tiempo, esto se aprecia en la Figura 3. Como se nota de los resultados se trata de tutores con cierto grado de experiencias en cuanto a la educación a distancia y en cursos en línea.

Sobre los quehaceres que debe realizar un tutor virtual al iniciar un curso, la población encuestada nos manifestó lo siguiente.

En el Figura 4, se muestra que el $76 \%$ de los tutores afirma que siempre "planifica con tiempo su curso" y el $17 \%$ señala que casi siempre y aunque es una minoría existe un $7 \%$ de los tutores que a veces o casi nunca lo

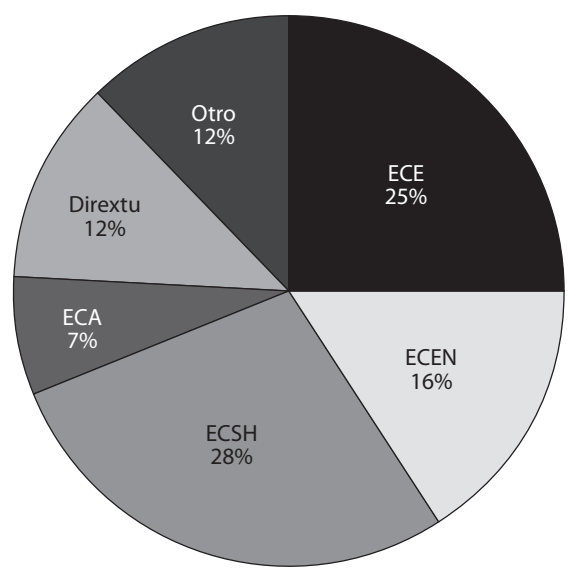

Figura 1. Escuela o dirección a la cual pertenecen los tutores. Fuente: elaboración propia. 


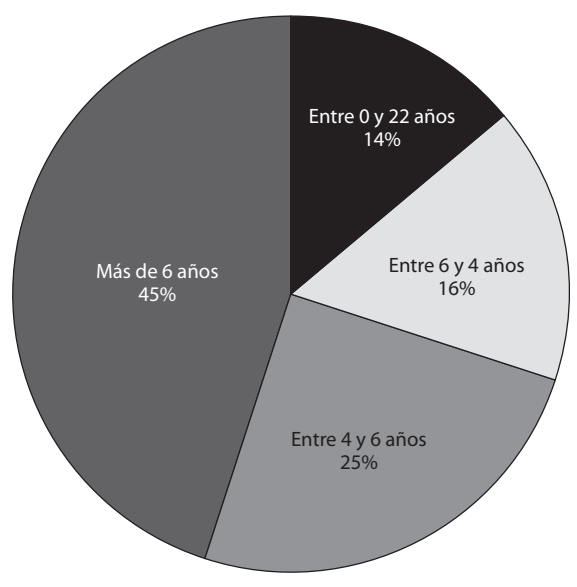

Figura 2. Tiempo de laborar en la UNED. Fuente: elaboración propia.

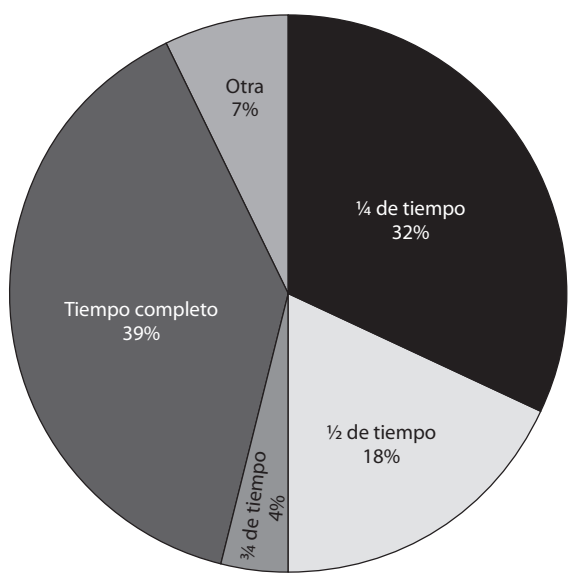

Figura 3. Jornada laboral como tutor de la UNED. Fuente: elaboración propia.

hace, situación que no debe presentarse ante un grupo de profesionales, esto por la importancia que tiene la revisión del curso y el cuidado por los detalles como lo son las actividades, materiales didácticos que se le presenta el estudiantado.

Las consignas son una lista de pasos por medio el cual el tutor le comunica al estudiante qué es lo que debe realizar para completar una actividad, esta debe ser tan clara de forma que oriente al estudiante a desarrollar la actividad de forma exitosa. Cuando se pregunta a los tutores si "redacta las instrucciones de las actividades de forma clara y concisa", el $60 \%$ afirma que siempre lo realiza y un $40 \%$ que casi siempre.

Sobre el aspecto que trata sobre "garantizar el acceso a la plataforma, ayuda técnica en el caso que las

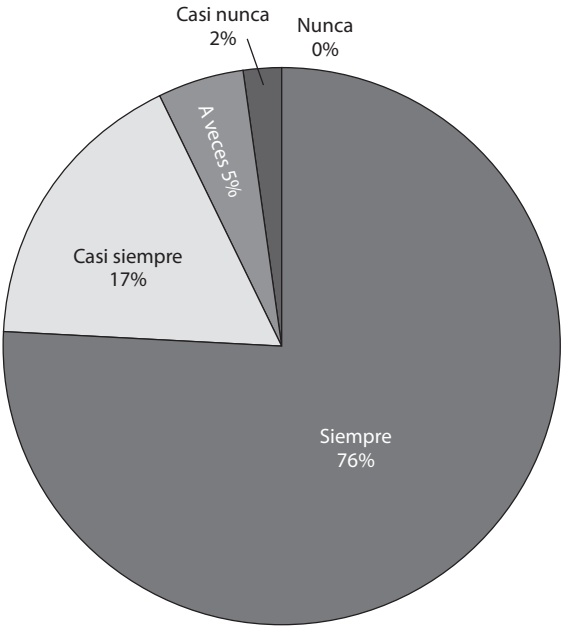

Figura 4. Planifica con tiempo su curso. Fuente: elaboración propia.

y los estudiantes lo requieran", prácticamente la mitad (51\%) de los tutores manifiestan que ellos siempre lo garantizan y un $35 \%$ que casi siempre (ver Figura 5). Si se analiza los resultados con respecto a la importancia que tiene brindar apoyo tanto pedagógico como técnico a los estudiantes, se puede pensar que este resultado preocupa ya que la mitad de las y los estudiantes puede estar quedando sin apoyo técnico para ingresar a la plataforma, si el estudiante no logra desenvolverse en la plataforma puede ser un factor que provoque desmotivación y hasta la deserción del curso.

Sobre el ítem que pregunta si "proporciona una orientación general del curso a los estudiantes", el $88 \%$

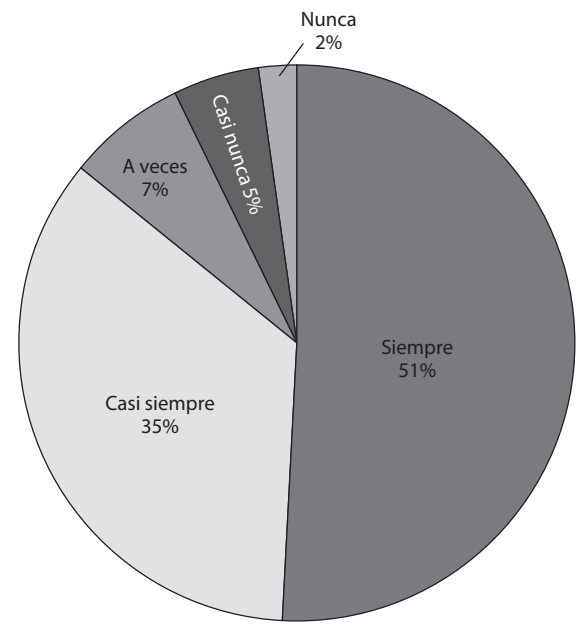

Figura 5. Garantiza el acceso a la plataforma. Fuente: elaboración propia. 
de los encuestados expresan que siempre han brindado orientaciones de curso o material adicional y un 12 $\%$ que casi siempre. En este caso la totalidad de los cursos deberían presentar la orientación del curso como insumo que permite al estudiantado organizarse en cuanto a las actividades y cronograma del curso.

Unos de los aspectos en que la mayoría coincidió fue en que el $95 \%$ manifiesta que siempre "brindan a las y los estudiantes una bienvenida al curso" y un $5 \%$ que casi siempre la brinda. Este dato es relevante ya que es parte de mejorar la dinámica al inicio del curso y que el estudiante conozca al tutor facilitador.

Se destaca en el Figura 6 que el $82 \%$ de los tutores concordaron en que ellos siempre "brindan las normas de las netiquetas a sus estudiantes" y un $12 \%$ pronuncia que casi siempre. Es importante mencionar que este tipo de normas, relativas a la adecuada interacción y uso de espacios de comunicación por parte de los estudiantes, permite desde el inicio del curso evitar problemáticas por el mal uso de la expresión escrita, entre otras recomendaciones.

En Figura 6 se observa que el $30 \%$ de los tutores expresan que casi siempre "realiza algún diagnóstico inicial al curso" y un $23 \%$ que siempre lo realiza, en cuanto a este aspecto las respuestas obtenidas reflejan que gran parte de los tutores no realizan siempre un diagnóstico inicial para conocer los conocimientos previos de sus estudiantes, lo que significa que al finalizar el curso tampoco tendrán un punto de comparación sobre cuál fue el aprendizaje de sus estudiantes.

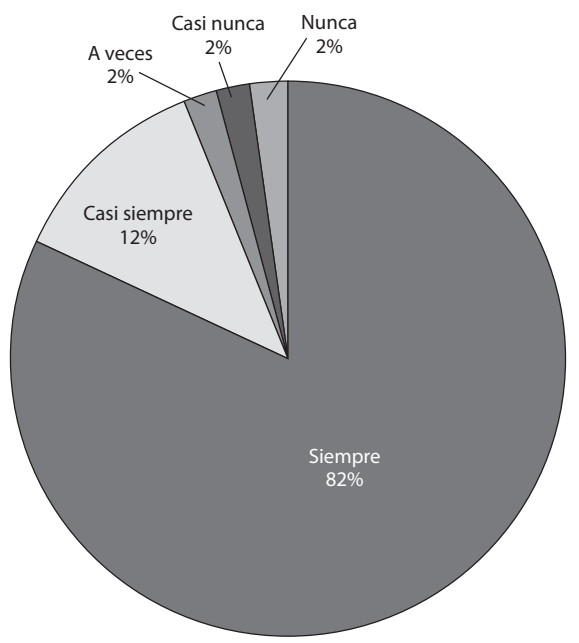

Figura 6. Ofrece a sus estudiantes las normas de las netiqueta.

Fuente: elaboración propia.
Además, el diagnóstico inicial permite al tutor virtual adaptar el curso a los participantes para mejorar la dinámica y propiciar el éxito en el mismo.

Las actividades exploratorias en cursos en línea se refieren al uso del foro, correo interno, envío de tareas de prueba, entre otras herramientas que se utilizarán en la clase que se imparte, de forma que estas se conozcan previamente a convertirse en actividades evaluadas. Esta práctica beneficia principalmente a las y los estudiantes que son nuevos en la modalidad a distancia y en cursos en línea, o bien para aquellas y los estudiantes con bajas o nulas competencias tecnológicas. Ante la consulta relativa a si "confecciona actividades exploratorias de la plataforma para que las y los estudiantes se familiaricen con el entorno virtual", el $61 \%$ expresa que siempre lo realizan y seguidamente un 21 $\%$ que casi siempre, porcentaje que no es satisfactorio si se piensa qué pasa con aquellas y los estudiantes que nunca han tenido una experiencia con cursos en línea, los resultados se muestran en el Figura 8.

En cuanto a la pregunta sobre si "se brinda un foro o espacio para que las y los estudiantes se presenten", podríamos decir que en su totalidad respondieron afirmativamente, esto porque el $95 \%$ menciona que siempre lo hace y un $5 \%$ que casi siempre. Esta actividad además de acercar a las y los estudiantes permitir que se conozcan aunque sea en la virtualidad, beneficia la ejecución de futuros foros académicos.

Lo mismo sucede con la respuesta en cuanto a si "proporciona espacios de comunicación y consultas en su curso", en este caso el $98 \%$ manifiesta que siempre lo hace y el $2 \%$ que lo hace casi siempre.

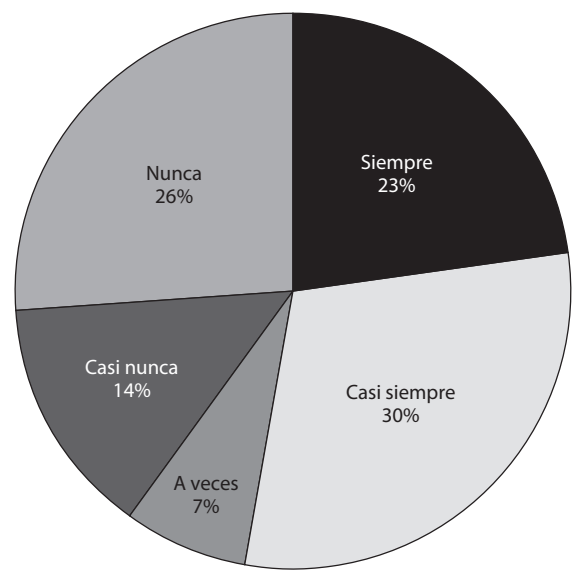

Figura 7. Realiza un diagnóstico inicial al curso. Fuente: elaboración propia. 


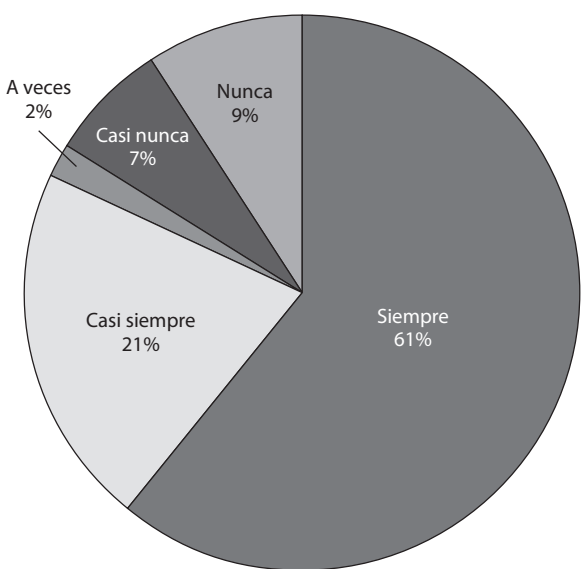

Figura 8. Brinda actividades exploratorias de la plataforma. Fuente: elaboración propia.

Con el fin de conocer la forma en que los tutores de la UNED desarrollan el curso se consultaron los siguientes aspectos.

Se consultó al tutor si "cumple usted con todas las fechas establecidas" y todas las respuestas fueron afirmativas, entre ellas un $79 \%$ menciona que siempre lo hace y un $21 \%$ que casi siempre. Cuando se habla de fechas establecidas estas tienen que ver con visualización de semanas o temas, apertura y cierre de actividades, así como la entrega de calificaciones, retroalimentación o atención del curso en general.

Una práctica importante en un curso en línea es el seguimiento y monitoreo de los estudiantes, esto beneficia a las y los estudiantes que puedan estar rezagados o bien que estén perdiendo actividades importantes

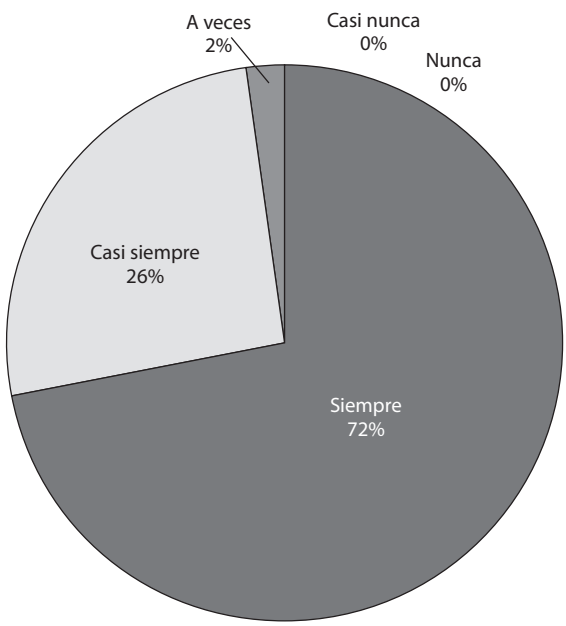

Figura 9. Brinda retroalimentación constante a los estudiantes. Fuente: elaboración propia. durante el curso. En el ítem que pregunta si "da seguimiento e incentiva a las y los estudiantes para que participen en las actividades" el $72 \%$ expresa que siempre lo realiza y el $28 \%$ que casi siempre.

En el Figura 9 se presenta el resultado a la consulta sobre si "brinda retroalimentación constante a los estudiantes" este resulta uno de los quehaceres indispensables en el rol del tutor y aunque la respuesta que se obtuvo fue de un $72 \%$ que expresan siempre hacerlo, hay un $28 \%$ de los tutores que aceptan hacerlo casi siempre, lo que significa que en ocasiones no lo hacen y un $2 \%$ de que a veces lo hace, es aquí donde surge la interrogante ¿cómo pretende un tutor enseñar a sus estudiantes sin retroalimentar? Ya que esta es una de las funciones básicas de todo docente.

Sobre la "entrega las calificaciones de las actividades en un tiempo prudente" todos afirman hacerlo siempre lo que representa un $68 \%$ y el $32 \%$ restante manifiesta que casi siempre lo hace.

De igual forma sucede cuando se preguntó si "está atento a las consultas de las y los estudiantes (por medio del correo interno y foro de consultas)" el $91 \%$ responde que siempre lo hace y el $9 \%$ que casi siempre.

Sobre el ítem que consulta si "utiliza instrumentos para evaluar las actividades" el $82 \%$ de los encuestados aseguran que siempre lo hacen y un $10 \%$ responde que casi nunca, seguidamente un $8 \%$ dice hacerlo casi siempre, tal como se muestra en el Figura 9. Estos resultados reflejan que un $18 \%$ de los tutores califican a sus estudiantes subjetivamente y no brinda previamente una lista de criterios a evaluar sobre las actividades, esto con el fin de que las y los estudiantes sean evaluados de una forma más objetiva, situación que debe evitarse porque el estudiantado tiene el derecho de conocer previamente cuáles son los criterios con los que se le va a evaluar su trabajo.

Al preguntar a los tutores si "ofrece documentos que informen y orienten las actividades a realizar en cada uno de los módulos, temas, unidades o semanas" el $82 \%$ revela que siempre lo hace y el $14 \%$ responde que casi siempre, mientras que el $2 \%$ menciona que a veces. El uso de este tipo de documento orientador es el que se ha generalizado en la UNED con el nombre de ruta de aprendizaje, aunque también se le denomina instrucciones, agenda o programación de la semana o módulo, documento que tiene aspectos generales como la evaluación, fechas de entrega y objetivos por 


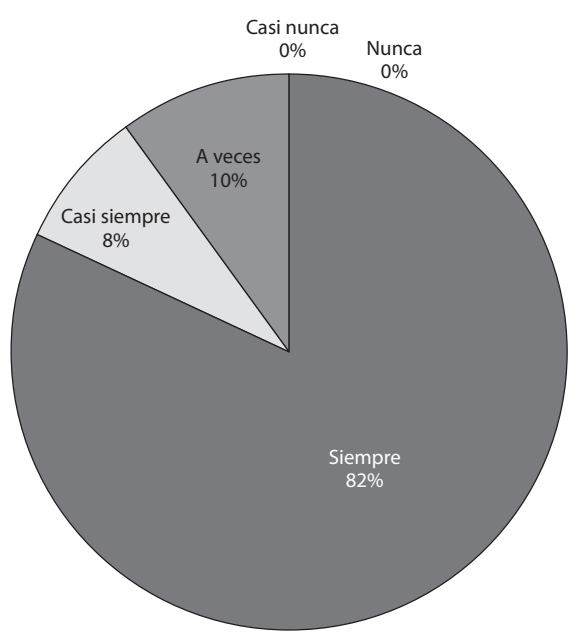

Figura 10. Utiliza instrumentos para evaluar las actividades. Fuente: elaboración propia.

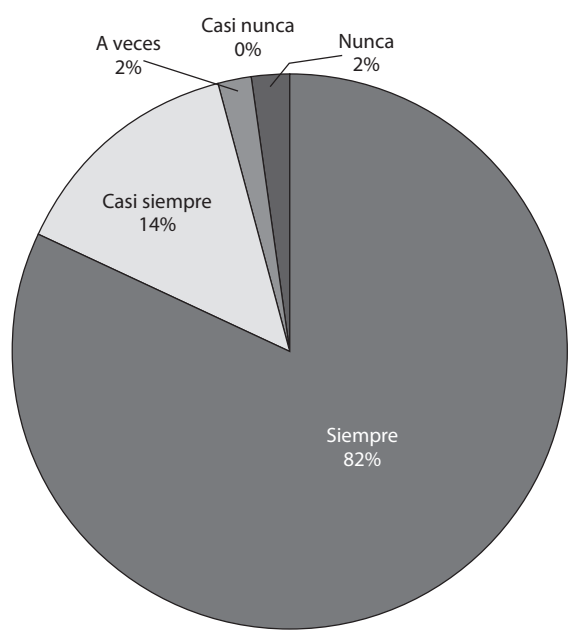

Figura 11. Ofrece documentos que informen y orienten las actividades. Fuente: elaboración propia.

cumplir, en el Figura 11 a continuación se pueden visualizar dichos resultados.

Al preguntar si "utiliza actividades interactivas, innovadoras y que promuevan el trabajo cooperativo y la autonomía", se nota que la respuestas afirmativas disminuyen significativamente ya que la mayoría (54\%) acepta utilizar este tipo de actividades casi siempre y solamente un $30 \%$ afirma siempre utilizar actividades interactivas e innovadoras (ver Figura 12), lo que refleja que en la mayoría de las ocasiones las y los estudiantes deben realizar actividades tradicionales y con carencias que incentiven el cooperativismo y la autonomía, aspectos fundamentales dentro del perfil de los estudiante de una universidad a distancia.

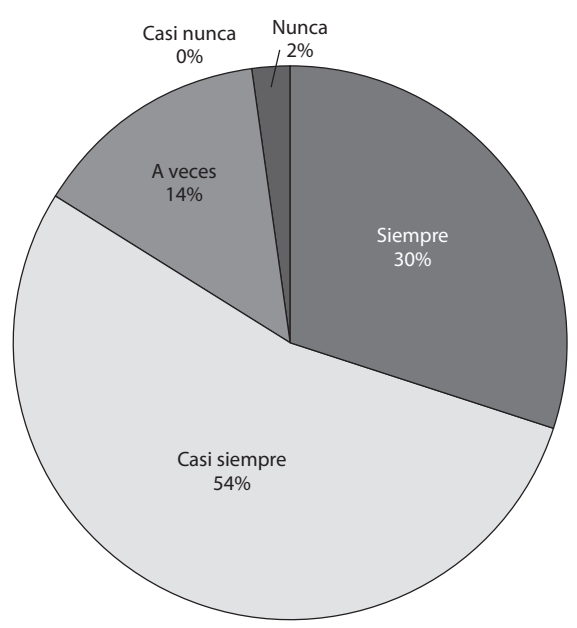

Figura 12. Utiliza actividades interactivas, innovadoras y que promuevan el trabajo cooperativo y la autonomía. Fuente: elaboración propia.

Al consultarle a los entrevistados si "mantiene comunicación constante con sus estudiantes" el $77 \%$ expresó que siempre lo hace y $23 \%$ que casi siempre, lo que refleja que mayormente hay buena comunicación entre el tutor y sus estudiantes.

Sobre aspectos relacionados con la parte de evaluación que debe tener todo curso, se preguntó a los tutores si "analiza los resultados obtenidos con respecto a los objetivos propuestos" y el $65 \%$ expresa que siempre lo hace, mientras que $26 \%$ manifiesta hacerlo casi siempre, un $7 \%$ lo realiza a veces y un $2 \%$ nunca lo hace, tal como se muestra en el Figura 13. En este caso, dados los resultados obtenidos, es necesario manifestar

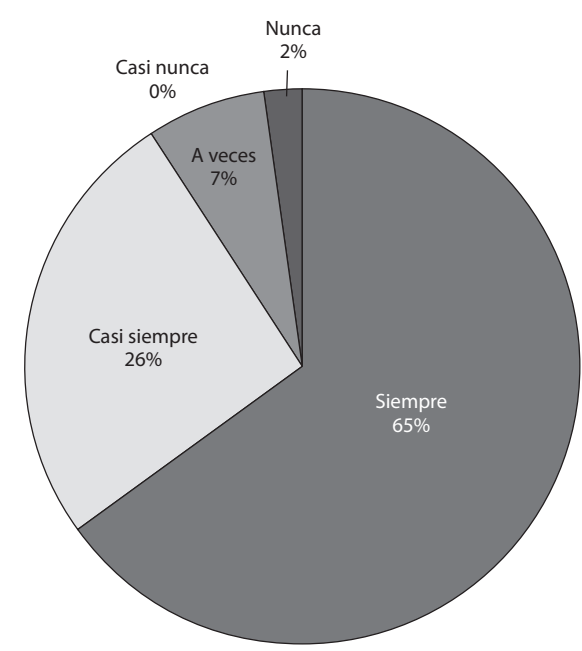

Figura 13. Analiza los resultados obtenidos con respecto a los objetivos propuestos. Fuente: elaboración propia. 


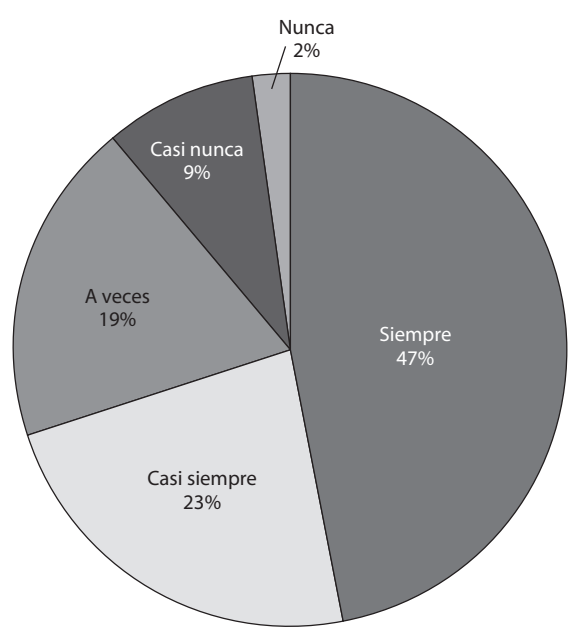

Figura 14. Realiza cierres o conclusión de las actividades. Fuente: elaboración propia.

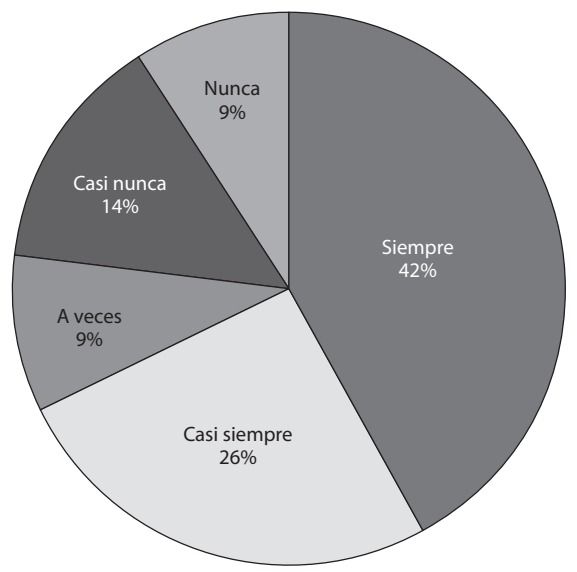

Figura 15. Realiza una evaluación final del curso. Fuente: elaboración propia.

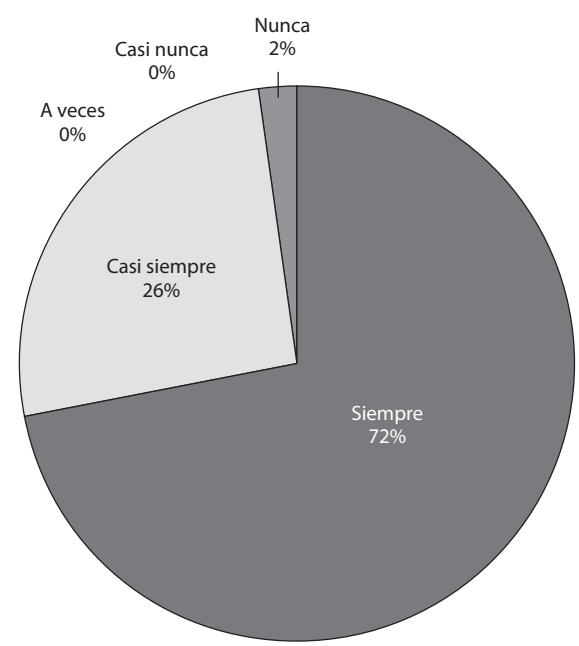

Figura 16. Actualiza constantemente su curso. Fuente: elaboración propia. que debería existir mayor análisis de los resultados que permita hacer los ajustes pertinentes en cada oferta del curso.

El cierre o conclusión de una actividad permite la retroalimentación para el estudiante, además permite que el tutor evidencie la presencia en el curso (ver Figura 14). En relación a este tema se pregunta a los tutores si "realiza cierres o conclusión de las actividades" las respuestas afirmativas nuevamente volvieron a disminuir ya que solamente el $47 \%$ de los tutores expresan que siempre lo hacen, mientras un $23 \%$ aceptó realizarlo casi siempre, un $19 \%$ dijo hacerlo a veces, un $9 \%$ afirma que casi nunca y hasta hubo quienes confirmaron nunca emplearlo (2\%).

Relacionado a la evaluación final que se deber realizar al culminar un curso se realizaron las siguientes consultas.

La evaluación final del curso permite que el facilitador o bien la persona que la aplica detecte carencias en cuanto a las actividades y la misma labor del tutor, a la vez que se pueden aplicar cambios para las siguientes ofertas. En el Figura 15 se muestran los resultados al consultarle al tutor si "realiza una evaluación final del curso, para verificar la percepción de los estudiantes", solamente el $42 \%$ de ellos pronuncian que siempre lo hacen y el resto expresa que casi siempre $26 \%$, casi nunca $14 \%$, a veces un $9 \%$ y nunca $9 \%$.

La actualización constante del curso orienta hacia la calidad del mismo, al preguntarle a los tutores si "actualiza constantemente su curso" el $72 \%$ responde que siempre lo hace, un $26 \%$ que casi siempre lo hace y un 2 $\%$ que nunca lo realiza, con estos últimos resultados surge la siguiente interrogante, en el Figura 16 se muestran dichos resultados, con esto surgen interrogantes como: ¿qué sucede con esos cursos que nunca son actualizados? Aunque es solamente un $2 \%$ de la población entrevistada, estos son docentes que trabajan en la UNED, una universidad que tiene como objetivo preparar a sus estudiantes para el futuro y brindar la mayor calidad en su oferta educativa, pero ¿se está monitoreando si se realizan las actualizaciones pertinentes a los cursos?

Al consultar a los tutores si "informa a sus autorizadas de forma objetiva sobre los resultados del curso", se puede observar en la Figura 17 que un $72 \%$ expresa hacerlo siempre, un $16 \%$ casi siempre, un $12 \%$ dice hacerlo a veces, casi nunca un 5\% y nunca 7\%, en el Figura 16 se muestra dicho comportamiento. La importancia 


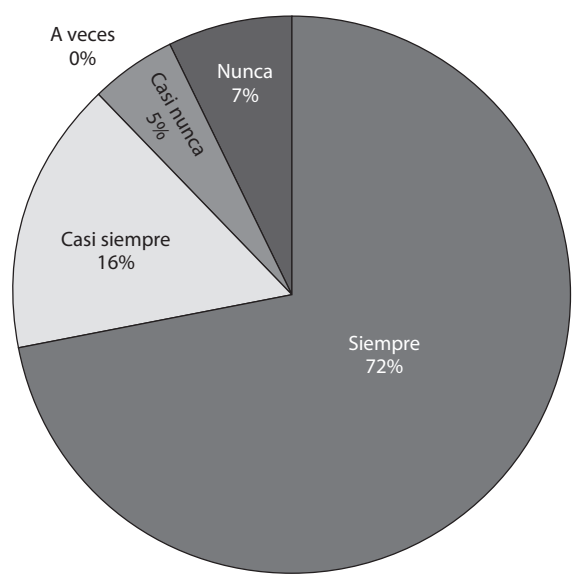

Figura 17. Informa a sus autorizadas de forma objetiva sobre los resultados del curso.

Fuente: elaboración propia.

de informar sobre los resultados del curso a las autoridades, en este caso a encargados de cátedra o programa, permite la toma de decisiones y correcciones oportunamente.

Para finalizar la encuesta se les preguntó a los tutores aspectos relacionados con la preparación profesional de cada uno de ellos, en cuanto a este tema las respuestas fueron las siguientes.

Sobre la consulta relativa a "el apoyo en cuanto a la capacitación que le ofrece la institución para su desempeño como tutor de cursos en línea", el $45 \%$ considera que es muy buena, el $27 \%$ expresa que es excelente y hay $28 \%$ que las considera entre Bueno (23\%) y Regular (5\%).
También se le preguntó a los tutores si “ha recibido capacitaciones de parte del Centro de Capacitación en Educación a Distancia (CECED)" y el $95 \%$ sí y un 5\% que no, situación que hay que revisar ya que se pretende que todo docente que esté atendiendo a estudiantes se encuentre capacitado.

Como complemento a la pregunta anterior se solicitó a los tutores señalar cuáles son los cursos en que ha participado y tal como se muestra en el Figura 18 la mayoría ha llevado el curso Diseño y Organización de cursos en línea y Pedagogía Universitaria para la Educación a Distancia.

Seguidamente se les preguntó a los tutores: ¿Considera usted que la(s) capacitación(es) cursada(s) es suficiente para su correcto desempeño en la tutoría virtual y cumplir con el rol un orientador para el estudiantado? La figura 18 muestra que el $50 \%$ considera que sí es suficiente pero el otro $50 \%$ considera que no es suficiente, lo que refleja que si se analiza con cuidado estos datos y los comparamos con la respuestas del ítem anterior que pregunta a los entrevistados sobre cómo era "el apoyo en cuanto a la capacitación que le ofrece la institución para su desempeño como tutor de cursos en línea", se nota que hay una contradicción porque anteriormente la mayoría calificó ese apoyo en cuanto a la capacitación como muy bueno pero ahora la mitad de esos mismos entrevistados consideran que la capacitaciones que han recibido no son suficiente para cumplir correctamente el rol como un tutor virtual (ver Figura 19).

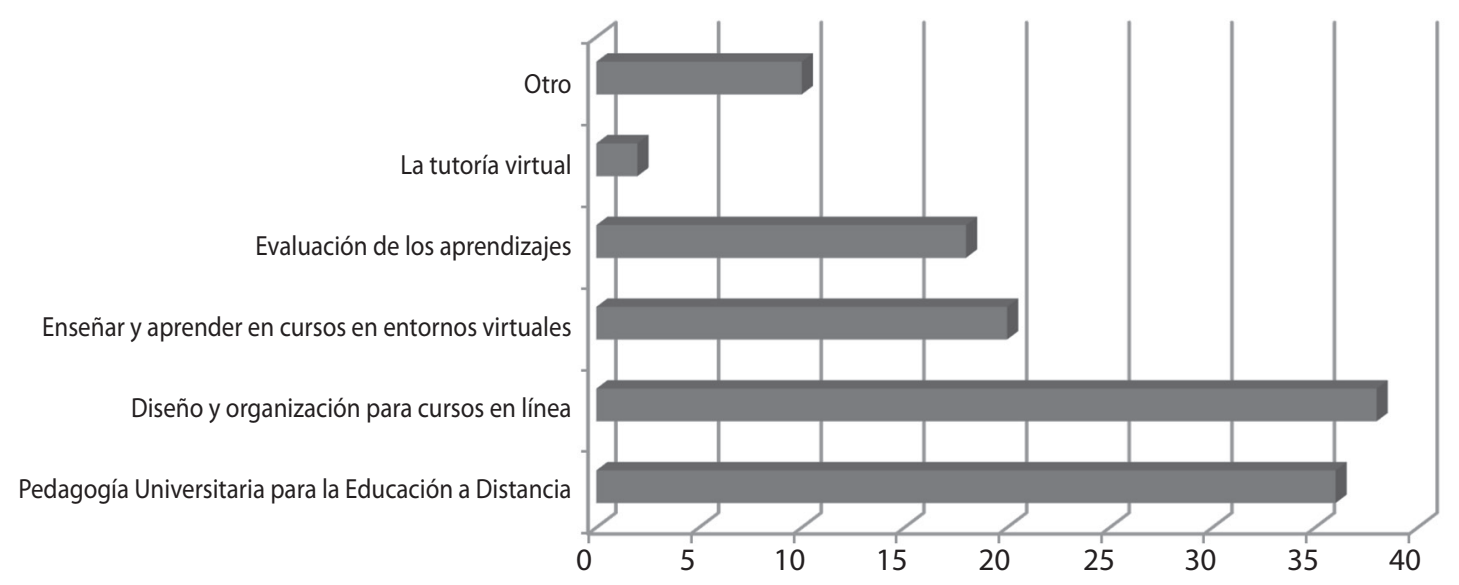

Figura 18. Tipos de capacitaciones recibidas. Fuente: elaboración propia. 


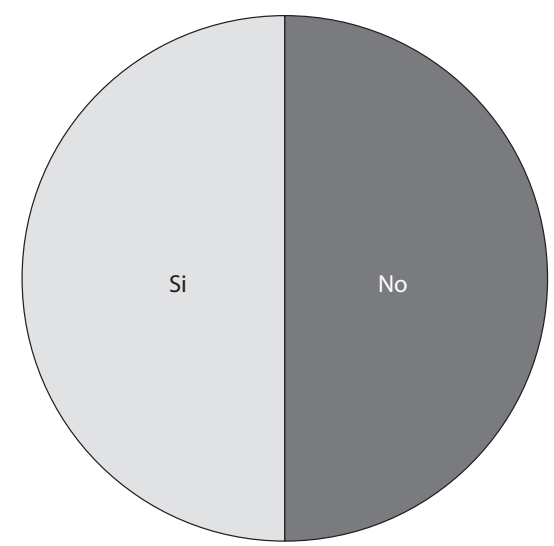

Figura 19. ¿Son suficientes las capacitaciones que ha recibido para cumplir con su rol como tutor virtual? Fuente: elaboración propia.

\section{CONCLUSIONES}

Un $17 \%$ de los tutores señala que no realiza la planificación previa del curso con tiempo, este porcentaje aunque es bajo, preocupa por la importancia que tiene la preparación de las actividades, material didáctico entre otros detalles importantes de un curso en línea.

El hecho de garantizar el acceso al curso en línea por medio de ayuda técnica y seguimiento debe ser vigilado ya que no todos los tutores realizan esta práctica que permite un mejor aprovechamiento del recurso virtual. Es recomendable uniformar esta práctica al inicio de un curso en línea con el fin de minimizar la deserción. Llama la atención que no todos los tutores tienen como práctica estandarizada proporcionar el programa del curso como documento orientador desde el inicio del curso, dado que este permite una mejor gestión y organización del tiempo por parte del estudiantado.

Además, no todos los tutores encuestados proporcionan las normas de la netiquetas para el inicio del curso, pese a que la institución tiene dos recursos sobre esta temática elaborados a la medida para los cursos en línea con el fin de preparar a las y los estudiantes sobre cómo se trabaja dentro de los entornos virtuales. Adicional a proporcionar la documentación importante al inicio del curso, es importante la actividad exploratoria en cuanto al uso de diferentes espacios de comunicación, como el foro, correo interno y envío de tareas en un curso en línea beneficia al inicio de un curso, no todos los tutores la aplican al inicio del curso, esto puede afectar a las y los estudiantes que son nuevos en los cursos en línea o bien que poseen bajas competencias tecnológicas.

Preocupa el hecho de que no todos los tutores realizan un diagnóstico inicial en el curso, ya que se trata de una actividad que permite conocer el nivel de las y los estudiantes y adaptar aspectos del curso para que se adapte mejor a los participantes.

Sobre el seguimiento e incentivo para la participación de las y los estudiantes en actividades, destaca el hecho que no todos los tutores lo aplican como regla general, esta es una función clave del tutor de cursos en línea, por cuanto permite detectar a estudiantes rezagados o bien que estén perdiendo actividades valiosas. Precisamente el uso de anuncios o correos personalizados por medio de la plataforma pueden motivar a una mayor participación en las actividades.

Llama la atención el resultado sobre el uso de los instrumentos de evaluación para las actividades de los cursos, pues no todos los tutores la utilizan, de hecho el $10 \%$ señala que casi nunca las utilizan. El uso de este tipo de instrumentos permite orientar al estudiante sobre la entrega que debe realizar y permite al facilitador valorar más objetivamente las actividades y realimentar de forma más detallada. En la UNED se tienen documentación de apoyo para tal efecto.

En cuanto al análisis de los resultados obtenidos con respecto a los objetivos propuestos, se obtiene de los encuestados que no todos desarrollan su curso enfocado en cumplir lo propuesto en el programa o diseño curricular del curso, esto evidencia que muchas veces el curso no tiene ajustes a lo largo del tiempo en cada oferta. Si se analizan el cumplimiento de los objetivos existe la oportunidad de realizar ajustes en las actividades, contenidos y evaluación propuesta.

Por los resultados obtenidos ante la consulta sobre si los tutores realizan cierres o conclusión de las actividades, se denota que no todos lo realizan, este tipo de labor permite una realimentación final de las actividades, colabora también con una mejor presencia y comunicación por parte del facilitador del curso.

Sobre la evaluación final del curso se constata que una reducida parte de la población aplica la evaluación final del curso, como proceso que permite una retroalimentación de las y los estudiantes hacia el tutor, en cuanto a la pertinencia de la evaluación del curso, las actividades, el material de estudio o los contenidos y 
en general el actuar del tutor como facilitador. El hecho de no aplicar este valioso proceso limita un curso de la mejora continua.

En cuanto a la actualización de los cursos existe un $2 \%$ de los tutores encuestados que no realizan el proceso y un $26 \%$ que señala que casi siempre lo hace, por tanto debe existir supervisión de aquellos cursos que no sufran mejora continua, esto puede estar relacionado con la falta de evaluación final por parte del curso.

En cuanto a la evaluación final del curso y su actualización, los tutores expresan que un $7 \%$ no informa del todo a las autoridades o a la coordinación sobre los resultados obtenidos. Esto puede evidenciar una falta de control hacia la labor de los tutores, pues pareciera que la iniciativa nace de ellos y no como directriz.

Sobre las capacitaciones que ofrecen el CECED, se destaca que un bajo porcentaje ha sido parte del curso que trata sobre la Tutoría virtual y sobre la Evaluación de los aprendizajes, esto se puede relacionar con algunas carencias detectadas sobre las funciones del tutor $y$ la evaluación.

\section{Recomendaciones para futuras investigaciones}

Con miras de ofrecer el servicio que las y los estudiantes de la UNED de Costa Rica se merecen, se recomienda continuar y ampliar este estudio para obtener las bases necesarias que fundamenten las decisiones que se requieran para fortalecer el cuerpo docente con el que cuenta la universidad.

De acuerdo con lo anterior se plantean las siguientes interrogantes:

- Si se entrevista a las y los estudiantes de estos profesores ¿existirá la misma percepción en cuanto a la forma que estos atienden los cursos?
- También se debe indagar por qué no se realiza como norma la evaluación final del curso, así como el envío de dicha información a los encargados de los cursos o asignaturas.

- $\quad$ Si existe documentación institucional sobre evaluación de los aprendizajes y uso de instrumentos de evaluación, por qué algunos tutores no la utilizan en los cursos para las actividades planteadas.

\section{REFERENCIAS}

Arboleda, N. y Rama, C. (2013). La educación superior a distancia y virtual en Colombia: nueva realidades. Recuperado de: http://virtualeduca.org/documentos/observatorio/ la_educacion_superior_a_distancia_y_virtual_en_colombia_nuevas_realidades.pdf

Delauro, M. (2011). Nuevos escenarios, nuevos roles docentes y nuevas competencias. Unidad 1-a, La tutoría en ambientes virtuales de aprendizaje. Virtual Educa.

Domínguez, J. y Rodríguez, J. (Eds.) (2013). La educación a distancia en el Perú. Recuperado de: http://virtualeduca. org/documentos/observatorio/la_educacion_a_distancia_en_peru.pdf

Ramírez, F y Rama, C. (Eds.) (2014). Los recursos de aprendizaje en la educación a distancia, nuevos escenarios, experiencias y tendencias. Recuperado de: http://www. virtualeduca.org/documentos/observatorio/2014/losrecursos-de-aprendizaje.pdf

UNED. (2012). Directrices para el trabajo en la plataforma. Recuperado de: http://recdidacticos.UNED.ac.cr/pal/ images/stories/Documentos_PAL/Directrices_trabajo_ plataforma_VF.pdf

UNED. (2010). Cómo diseñar y ofertar cursos en línea, consideraciones generales. Recuperado de: http://recdidacticos. UNED.ac.cr/pal/images/stories/Documentos_PAL/disenar_ofertar_cursos_linea.pdf

UNED. (2012). Reglamento General Estudiantil de la UNED. Recuperado de: http://www.UNED.ac.cr/academica/index.php/cidreb/cidi/normativa-universitaria/27-cidreb/ 228-reglamentos-area-estudiantil 